\title{
Deep Learning-enabled Detection of Acute Ischemic Stroke using Brain Computed Tomography Images
}

\author{
Khalid Babutain ${ }^{1}$, Muhammad Hussain ${ }^{2}$, Hatim Aboalsamh ${ }^{3}$, Majed Al-Hameed ${ }^{4}$ \\ Department of Computer Science, College of Computer and Information Sciences, King Saud University, Saudi Arabia ${ }^{1,2,3}$ \\ Department of Neurology, National Institute of Neuroscience, King Fahad Medical City, Saudi Arabia ${ }^{4}$
}

\begin{abstract}
Stroke is the second leading cause of death globally. Computed Tomography plays a significant role in the initial diagnosis of suspected stroke patients. Currently, stroke is subjectively interpreted on CT scans by domain experts, and significant inter- and intra-observer variation has been documented. Several methods have been proposed to detect ischemic brain stroke automatically on CT scans using machine learning and deep learning, but they are not robust and their performance is not ready for clinical practice. We propose a fully automatic method for acute ischemic stroke detection on brain CT scans. The system's first component is a brain slice classification module that eliminates the CT scan's upper and lower slices, which do not usually include brain tissue. In turn, a brain tissue segmentation module segments brain tissue from CT slices, followed by tissue contrast enhancement using the Extreme-Level Eliminating Histogram Equalization technique. Finally, the processed brain tissue is classified as either normal or ischemic stroke using a classification module, to determine whether the patient is suffering from an ischemic stroke. We leveraged the use of the pre-trained ResNet50 model for slice classification and tissue segmentation, while we propose an efficient lightweight multi-scale CNN model (5S-CNN), which outperformed state-of-the-art models for brain tissue classification. Evaluation included the use of more than 130 patient brain CT scans curated from King Fahad Medical City (KFMC). The proposed method, using 5-fold cross-validation to validate generalization and susceptibility to overfitting, achieved accuracies of $99.21 \%$ in brain slice classification, $99.70 \%$ in brain tissue segmentation, $87.20 \%$ in patient-wise brain tissue classification, and $90.51 \%$ in slice-wise brain tissue classification. The system can assist both expert and non-expert radiologists in the early identification of ischemic stroke on brain CT scans.
\end{abstract}

Keywords-Acute ischemic brain stroke; deep learning; convolutional neural network; CT brain slice classification; brain tissue segmentation; brain tissue contrast enhancement; brain tissue classification

\section{INTRODUCTION}

Globally, stroke is a leading cause of death, accounting for around 15 million deaths annually [1], [2]. Even in low-income and middle-income countries, stroke is a major cause of mortality, and in the Kingdom of Saudi Arabia (KSA), annual stroke incidence has increased to 29.8 per 100,000 [3]. Notably, $87 \%$ of all stroke incidents result from ischemic stroke, whereas the remaining $13 \%$ are hemorrhagic [4]. Andersen et al. [5] investigated 39,484 stroke patients, reporting that 35,491 (89.9\%) suffered from ischemic stroke and 3,993 (10.1\%) experienced hemorrhage stroke. Generally, stroke arises from the sudden interruption of blood flow to neuronal tissue; a blockage within blood vessels leads to ischemic stroke, while blood vessel rapture causes hemorrhagic stroke [2]. To manage ischemic stroke, anti-thrombolytic therapy (removing the blockage by clot breaking) and thrombectomy (removing the clot mechanically) are used, while decompression and blood pressure reduction are used for hemorrhagic stroke [2].

Diagnostic imaging is essential in routine clinical practice to confirm early-stage ischemic stroke. Computed Tomography (CT) is regarded as the front-line modality to evaluate patients with suspected stroke due to its accessibility and costeffectiveness, which is not the case with Magnetic Resonance Imaging (MRI) [6], [7]. Typically, suspected stroke patients are handled by emergency room physicians, and the condition is often misdiagnosed or diagnosed late due to difficulties in arranging urgent assessments with experienced neuroradiologists. This often negatively influences stroke management [8].

State-of-the-art methods in computer science are assisting clinicians and neurologists, including for the application of image processing techniques to digital medical images [9], [10], [11]. For example, Chung et al. [12] developed a system to detect hyperacute ischemic stroke in CT images, achieving an accuracy of $81 \%$ in classifying stroke and non-stroke images. Methodologically, the authors extracted ranklet features from pre-processed CT images and identified 23 important features for stroke detection, 8 of which were used to establish the prediction model. Guoqing et al. [13] developed a system based on asymmetric image patch classification to detect ischemic stroke signs on non-contrast CT images, achieving an accuracy of $76.84 \%$ on 108 stroke cases that trained radiologists did not detect.

Chin et al. [14] developed a CNN model for automatic ischemic stroke diagnosis. Model training and testing involved 256 patch images of size $32 \times 32$ extracted manually from CT images. Their system achieved testing and training accuracies of $92 \%$ and $97 \%$, respectively. Pereira et al. [15] used two CNNs, one with a 50/50 protocol and another with $75 / 25$, for training and testing with $300 \mathrm{CT}$ images (100 healthy, 100 ischemic, 100 hemorrhagic). Contrasting architectures were used, with the most effective results being 97.5\%, 100\%, and 99.1\% classification accuracies for hemorrhagic, ischemic, and healthy images, respectively, using the $75 / 25$ protocol on their second model. Anis et al. [16] applied deep transfer learning for ischemic stroke detection on CT images, using 400 images with data augmentation (specifically, horizontal flipping) to compare the results to ResNet50, GoogleNet, and VGG-16 pre- 
trained CNNs. Using 5-fold cross-validation, they reported that ResNet50, GoogleNet, and VGG-16 achieved 100\%, 99.4\%, and $92.2 \%$ accuracies, respectively, on their training set, while accuracies of $100 \%, 98.8 \%$, and $90 \%$ were reported on the validation set.

Gautam and Raman [17] developed a 13-layer CNN model to classify ischemic and hemorrhagic stroke. Their CT image dataset (300 healthy, 300 ischemic, 300 hemorrhagic) was preprocessed using quadtree-based multi-focus image fusion [18]. Two copies were created for each image, after which contrast adjustment was applied to the first and filtering to the second using a $3 \times 3$ averaging filter. The copies were fused and passed to the CNN model, which consisted of an input layer $(512 \times 512 \times 1)$ and two convolutional layers, each followed by a Rectified Linear Unit (ReLU) activation and max-pooling layer, two fully connected layers with a ReLU activation and dropout layer after the first fully connected layer, and a softmax classification layer. Two datasets were established, one containing stroke images only and another containing both stroke and healthy images, and an 80/20 data split protocol with 10 -fold cross-validation was applied in each case. They achieved $98.33 \%$ and $98.77 \%$ classification accuracies on the first dataset, respectively, whereas $92.22 \%$ and $93.33 \%$ were achieved for the second dataset. The model significantly outperformed fine-tuned AlexNet and ResNet50.

These results highlight the clinical value of computational techniques in stroke detection [19], [20]. However, there is room for improvement, which has caused researchers to leverage Deep Learning (DL) techniques. DL is a subfield of artificial intelligence wherein algorithms learn to make accurate predictions without explicit programming [9], [11], [19]. Convolutional Neural Network (CNN) is a biologically inspired DL paradigm that holds promise in diagnostic medicine due to its ability to outperform humans in image and speech recognition/translation tasks [21].

This research proposes a DL system for acute ischemic stroke classification on brain CT scans. The system determines whether a brain CT slice contains brain tissue, segments the brain tissue, enhances the contrast of the segmented brain tissue, and identifies signs of acute ischemic to determine stroke incidence. CNN-based techniques are adopted in the first two tasks, whereas an efficient multi-scale CNN model - the 5-Scale CNN model (5S-CNN) - is proposed to resolve difficulties associated with distinguishing between normal/abnormal brain regions. A dataset from King Fahad Medical City (KFMC) containing over 130 annotated patient records is used to design, develop, and validate the system, and cross-validation is performed to evaluate the CNN models.

\section{PROPOSED METHOD}

The main technical objective of this research is to develop a robust and intelligent method for the diagnosis of ischemic stroke on brain CT scans, which will assist the clinical decision-making of neurologists. In routine clinical practice, brain CT scans are manually interpreted by professionals, expert operators, or both. This process involves the manual scanning of each slice of the patient's brain CT scan for the presence of stroke. Each patient's CT scan contains 35 to 45
CT slices on average (based on the collected dataset). Manual scanning also includes manual adjustment and enhancement of the contrast of the scanned slice for better visualization.

To formulate such a process, let $C=\left\{S_{i}\right\}_{i=1}^{n}$ represent a patient's CT scan, which consists of $n$ slices $S_{i}$ of size $512 \times 512 \times 1$, as shown in Fig. 1 .

Ischemic stroke can appear in any slice within a patient's CT scan; it can also appear at any location in the brain tissue within a slice. Ischemic stroke changes the texture of the affected region of brain tissue, as indicated by comparing the left panel of Fig. 2, which shows a normal brain CT slice, to the right panel, which shows an example of acute ischemic stroke.

Fig. 2 reflects the fact that ischemic stroke can affect any region of the brain tissue. Additionally, the affected area can have any regional size. The affected area becomes darker in texture as the time from its occurrence increases [22].

To determine whether a patient is experiencing an ischemic stroke, it is necessary for a sign of the ischemic stroke to appear on at least one slice of the patient's CT scan. Therefore, each slice must be processed individually, which can be categorized as a classification problem. In any given brain CT slice, there are usually parts that are irrelevant (e.g., scalp, skull, and unrelated background objects) because they do not contribute to the diagnosis. Such parts must be removed, enabling only the brain tissue region to be segmented and separated, which can be categorized as a segmentation problem. Additionally, certain slices in the upper and lower parts of the CT scan do not include brain tissue, and so these slices must be excluded before any processing occurs. This also is categorized as a classification problem, the objective of which is to identify whether a given CT slice contains brain tissue.

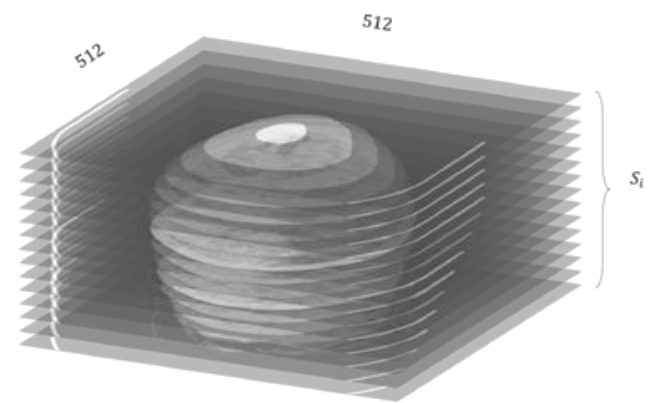

Fig. 1. Brain CT Scan of a Patient.
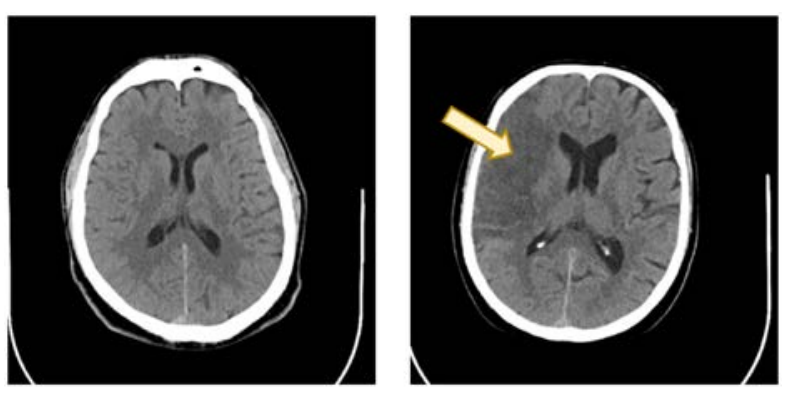

Fig. 2. Normal Brain CT Slice (Left) and Acute Tschemic Stroke (Right). 


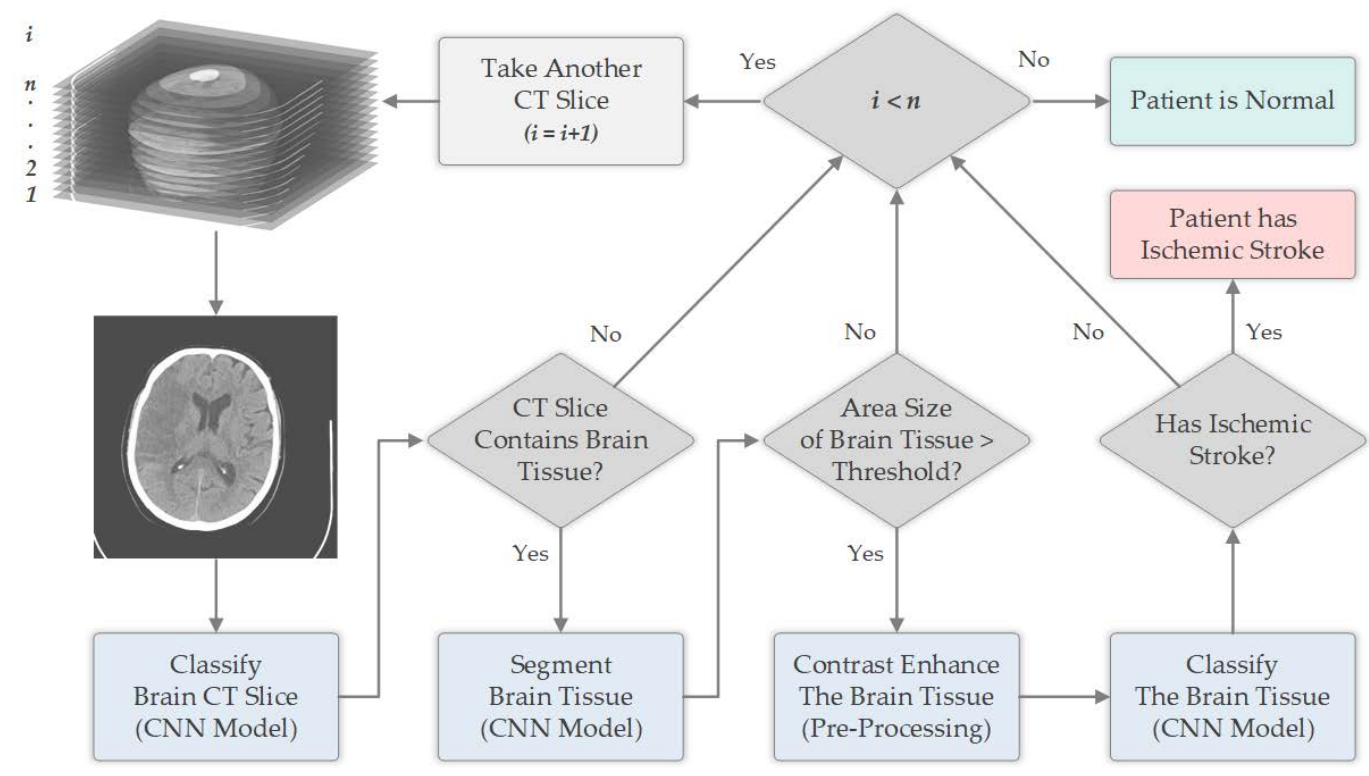

Fig. 3. The Architecture of the Proposed Method.

Given the above discussion, the development of an intelligent method for the automated diagnosis of a patient's brain CT scan involves four main components, which collectively process each slice of a given brain CT scan and systematically determine whether the patient is experiencing an ischemic stroke. As shown in Fig. 3, these components are the Brain CT Slice Classification Model, Brain Tissue Segmentation Model, Brain Tissue Contrast Enhancement Component, and Brain Tissue Classification Model.

The proposed method begins by reading an input CT slice to determine whether the slice should be considered for processing. In any brain CT scan, it is common for the first and last few slices not to contain brain tissue; these slices must be removed prior to any further processing. Also, since some of these CT slices do not contain brain tissue, despite having textural similarities to slices with brain tissue (see Fig. 4), it is necessary to leverage a classification model to determine whether a given slice contains brain tissue.

Before developing this model, a dataset was prepared containing labeled images of CT scan slices with and without brain tissue. Fine-tuning, training, and evaluation of the pretrained networks were performed. Fine-tuning involves updating each model's input layer to match the size of brain CT slices, which are fixed at $512 \times 512 \times 1$. Also, the first convolutional layer's kernels, which are 3-dimensional (spatial $\times$ depth) kernels $k(x, y, 3)$, are updated to 2-dimensional kernels $k^{\prime}(x, y)$ using the mapping $K: k(x, y, 3) \rightarrow k^{\prime}(x, y)$ such that

$k^{\prime}(x, y)=K(k(x, y, 3))=\frac{1}{3} \sum_{d=1}^{3} k(x, y, d)$

The final classification layer was also replaced with a new classification layer consisting of two neurons. This is because CT slice classification is a two-class problem (i.e., slice with/without brain tissue). Fig. 5 shows the concept of updating a pre-trained network to be re-trained for brain CT image classification. Training and evaluation included the use of K-fold cross-validation, which increased reliability and generalization and enabled the selection of the model with the best results.

After confirming the existence of brain tissue within the input CT slice, the brain tissue segmentation component detects and segments the brain tissue. In this process, irrelevant parts are removed and only the brain tissue is retained. It begins with an original input slice, as shown in Fig. 6(a). A trained semantic segmentation model is then applied, resulting in an image segmented by class, as in Fig. 6(b). Finally, morphological image analysis is implemented by maintaining the largest segmented group of pixels and filling its holes to obtain the segmented brain tissue [25], as shown in Fig. 6(c).
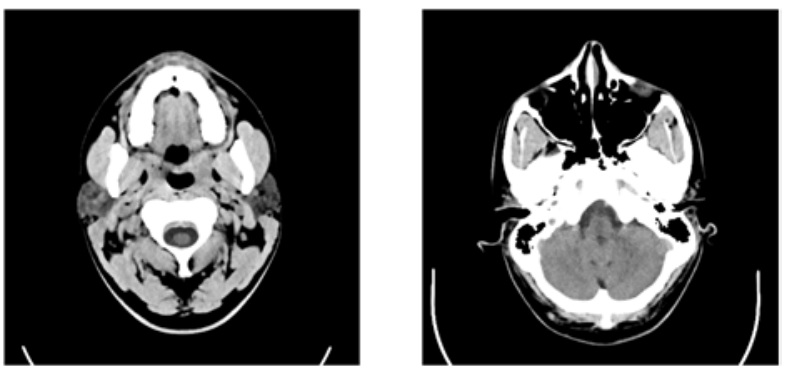

Fig. 4. CT Image with No Brain Tissue (Left) and CT Image with Brain Tissue (Right).

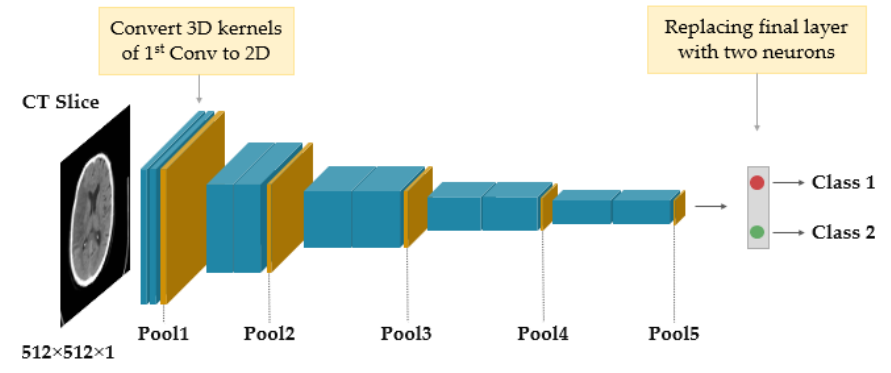

Fig. 5. Adopting a Pre-trained CNN Model for CT Slice Classification. 


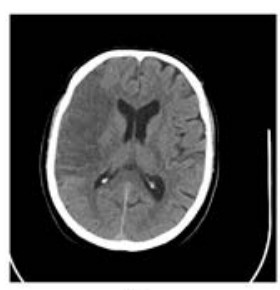

(a)

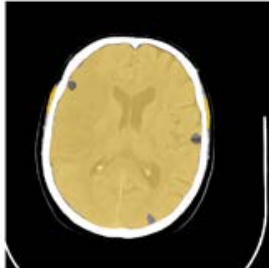

(b)

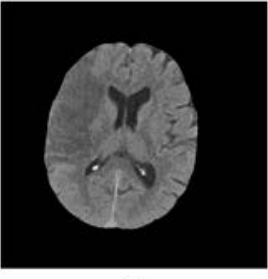

(c)
Fig. 6. Brain Tissue Segmentation: (a) Original Image, (b)

Applying Semantic Segmentation Model, and (c) Maintaining the Largest Segmented Group of Pixels and Filling its Holes.

DL-based segmentation methods have shown promising results for the segmentation of medical images [26], [27], [28]. The state-of-the-art DL-based framework, namely Fully Convolutional Networks (FCNs) [29], has been used for brain tissue segmentation. FCNs implement a pixel-wise classification process using a CNN model as a classification backbone, which classifies each pixel within an image and assigns it to a particular class, thereby resulting in an image segmented by class. We employed the FCN-8 architecture and tested the four widely-used state-of-the-art pre-trained CNN models as a classification backbone: AlexNet, GoogleNet, ResNet18, and ResNet50. As in brain CT slice classification, ResNet50 yielded the best segmentation results compared to the other models.

Before developing this model, an annotated dataset was prepared by manually annotating the pixels of each CT slice. Each pixel value in every image of this dataset represents a categorical label (either a brain tissue pixel or not). Fine-tuning involved updating the backbone model's input layer to take a CT slice of size $512 \times 512 \times 1$ as input and, in turn, updating the first layer's kernels from $3 \mathrm{D}$ to $2 \mathrm{D}$, as implemented for slice classification. In addition to this update, all pre-trained models were modified according to the FCN-8 up-sampling structure of the FCNs approach [29]. For training and evaluation, K-fold cross-validation was also used to select the most reliable and generalized segmentation model. Fig. 7 illustrates the concept of updating a pre-trained network according to the FCN-8 architecture to be re-trained for brain tissue segmentation.

Although the optimal segmentation model may lead to high segmentation accuracy, it is common for certain pixels or groups of pixels to be misclassified. This is shown in Fig. 6(b), where some pixels are classified as brain tissue while they are not, and vice versa. Therefore, post-processing is needed, which involves removing small connected components of pixels, and retaining the largest connected group of pixels (which usually represents brain tissue). This is followed by filling in the holes [30], [25] if there is a sufficient ischemic stroke identification area size. Based on recommendations from a medical team, the final segmented brain tissue must be sufficiently large in terms of its area to contribute to the identification of an ischemic stroke. Thus, to make decisions about whether to include or exclude the segmented brain tissue for further processing, the area of the segmented brain tissue (in pixels) is compared to a fixed threshold recommended by the medical team. This comparison excludes any insufficient and small, segmented brain tissue that has an area smaller than the fixed threshold.

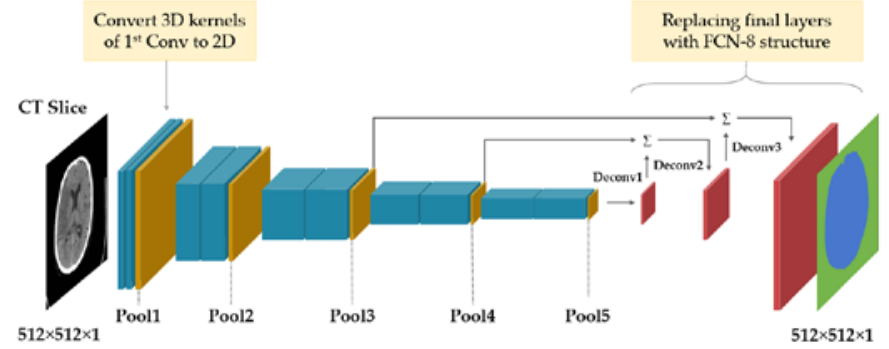

Fig. 7. Adjusting a Pre-Trained Network for Brain CT Image Ssegmentation using FCN-8 Structure.

Due to the low contrast of brain CT slices, multiple image contrast enhancement techniques have been proposed, particularly for medical images [31], [32], [33], [34]. These techniques are used to boost the interior details within brain tissues for visibility, classification, and ultimately interpretation. The Extreme-Level-Eliminating Histogram Equalization (ELEHE) method, proposed by Tan et al. [35], was developed mainly to improve ischemic stroke detection on brain CT images. ELEHE ensures that substantial differences in the distribution of the input CT image histogram are eliminated, resulting in a stretched histogram containing every intensity level value other than the unnecessary two extreme levels that result from regular Histogram Equalization (HE) [30].

Before enhancement, each slice is normalized by stretching the grey-level values within the range of 0 to 216. To enhance a CT slice using ELEHE, the first step calculates the Probability Density Function (PDF) of the slice's grey-level values. The next step involves eliminating the two extreme levels of the resulting PDF, thereby ensuring the maintainability of those levels while stretching the remaining grey levels. Following this, the Cumulative Density Function (CDF) is computed, after which a Transfer Function (TF) is applied to the values of the resulting CDF. Fig. 8 shows a brain CT image enhanced with ELEHE contrast enhancement.
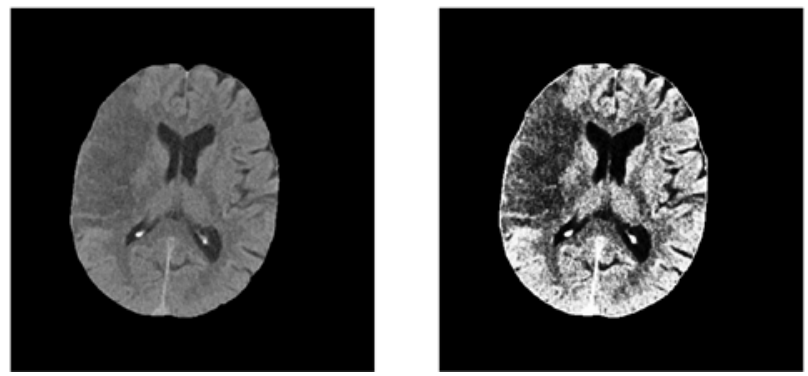

Fig. 8. Brain CT Image (Left) and Brain CT Image after applying ELEHE (Right).

After segmenting the brain region and applying contrast enhancement, the resulting brain tissue is classified to determine whether the CT slice is a case of normal or ischemic stroke, the latter of which indicates that the patient is suffering from ischemic stroke. If the classification of every processed slice of the CT scan is normal, then the patient is regarded as not suffering from ischemic stroke. Since there are two classes to consider (i.e., ischemic stroke or normal), the dataset preparation for this classification included labeled CT slices 
indicating an incidence of ischemic stroke or not. All slices with ischemic stroke incidence were labeled as acute, while slices with no stroke incidence were labeled as normal.

Ischemic stroke can manifest with any regional size and it can appear at any location within the brain tissue visualized by CT. Given this, a multi-scale analysis of brain tissue is needed to determine whether there is an instance of ischemic stroke. State-of-the-art pre-trained CNN models such as AlexNet, GoogleNet, ResNet18, and ResNet50 deal with only one scale, therefore the performance of these models is unsatisfactory. To overcome this issue, a lightweight multi-scale CNN model named the 5-Scale CNN model (5S-CNN) is proposed. After segmentation and contrast enhancement of the input CT brain tissue image, 5S-CNN uses a 5-branch architecture wherein each branch applies a different filter size to learn features at different scales.

5S-CNN consists of 77 layers, as shown in Fig. 9. Within this model, a total of 16 convolutional (Conv) blocks are used, where each block starts with a Conv layer followed by Batch Normalization (BN) and Rectified Linear Unit (ReLU) layers. The ReLU layers introduce non-linearity into the model in a very simple way of applying a thresholding operation to the pixels resulting from the $\mathrm{BN}$ layers, in which positive pixels are retained, and negative ones are assigned to zero. A maxpooling layer is positioned after each Conv block, resulting in a total of 16 pooling layers in the proposed model. The use of max-pooling layers enables the selection of only one pixel whose value is the highest compared to the other pixels within the pooling receptive field. This leads to the extraction of relevant features as well as a reduction in image size.

Within the first Conv layer of the proposed model, 16 filters with a size of $4 \times 4$ and stride of $2 \times 2$ are used, which down-samples the CT input image from $512 \times 512 \times 1$ to $255 \times 255 \times 16$. Thereafter, max-pooling with a size of $3 \times 3$ and stride of $2 \times 2$ is applied, which reduces the first Conv output size to $127 \times 127 \times 16$. It is then passed to 5 branches having three consecutive Conv blocks in each branch. The first Conv blocks in these branches contain 32 filters in each branch with sizes of $11 \times 11,9 \times 9,7 \times 7,5 \times 5$, and $3 \times 3$ in order to extract and learn features at different scales. After the first Conv block of each branch, two Conv blocks are used with 64 and 128 filters, respectively, of size $3 \times 3$ and stride $1 \times 1$; in these Conv blocks, a $3 \times 3$ filter size is used to reduce the number of learnable parameters as well as the model's computational cost. Additionally, each Conv block within each branch is followed by a max-pooling layer with a receptive field size of either $3 \times 3$ or $2 \times 2$ depends on whether the previous output feature map is odd or even in terms of its width and height.

At the end of each branch, a Global Average Pooling (GAP) layer is used to generate a channel descriptor for that branch. The resulting 5 GAP descriptors are combined using a concatenation layer and, in turn, for fusion, the output is used as an input to a Fully Connected (FC) block that has an FC layer with 256 filters, followed by a BN layer and ReLU layer, respectively. In the end, a single FC layer with two neurons and a softmax are used as a classifier. The number of neurons in this FC layer is targeted at the number of classes of interest (i.e., ischemic stroke or normal).

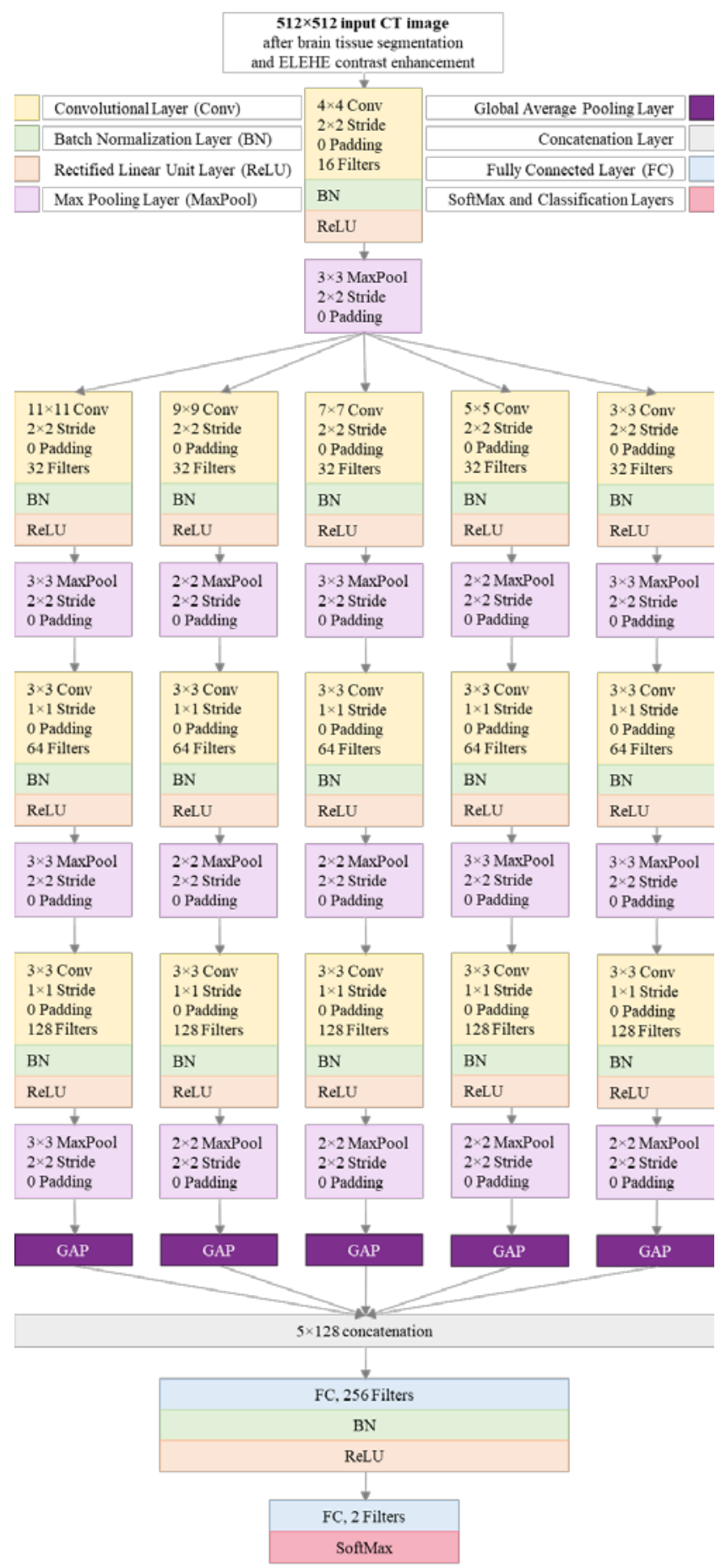

Fig. 9. The Proposed 5-Scale CNN Model (5S-CNN) Architecture for Ischemic Stroke Classification.

For comparison, the pre-trained models for tissue classification were fine-tuned using the approach adopted in the first and second components (i.e., CT slice classification and brain tissue segmentation). Specifically, the input layer of each pre-trained model was updated to match the DICOM brain CT image size of $512 \times 512 \times 1$. Additionally, the filters' weights for the first convolutional layer were updated from 3dimensional kernels to 2-dimensional kernels, which was achieved by taking the mean of each filter value across the 
depth dimension, as in (1). The final classification layer was replaced with new classification layers targeted at brain tissue classification.

The same training and evaluation procedures were performed on each model, including the 5S-CNN, and each segmented CT slice was contrast-enhanced using ELEHE before being passed as an input to any model. As in the first and second components, K-fold cross-validation was used to validate the reliability and generalization of each model.

\section{Dataset Collection, Annotation, And PREPARATION}

The dataset used to develop and validate the proposed method was collected from King Fahad Medical City (KFMC) under Institutional Review Board (IRB) approval with log number (17-031). The dataset contains brain CT scans from more than 130 patients, consisting of proven cases of both normal and acute ischemic stroke scans. The collection process involved compiling a shortlist from clinical and radiologic databases of patients who presented at emergency rooms or clinics between January 2015 and January 2018 with symptoms and signs of stroke. Revision for inclusion and exclusion of the selected patients was performed, in which scans with artifacts (e.g., motion and metal) and findings of hemorrhagic stroke were excluded. The remaining scans were included for analysis and modeling. During the medical team's annotation process, records of included patients were annotated and categorized into either normal or acute based on review findings. Each scanned record was further annotated by specifying the slices of the patient's CT scan that were affected by the ischemic stroke.

Dataset preparation for the Brain CT Slice Classification Model included a subset of 1,130 CT images selected randomly from 100 patients. The dataset contained 570 images labeled as Brain CT Slice from 50 patients, and 560 images labeled as Not Brain CT Slice from 50 patients. For the Brain Tissue Segmentation Model, another subset of the collected dataset was prepared that included 365 CT images from 18 randomly selected patients. This subset was manually labeled using MATLAB Image Labeler under the supervision of the medical team. Pixel-wise labeling was used to label each selected image, wherein each pixel in every image was labeled as either Brain Tissue or Not Brain Tissue. In the Brain Tissue Classification Model, the collected dataset included 63 patients with an acute diagnosis, having 300 CT images labeled as Acute. For data balancing and cross-validation, 62 patients with a normal diagnosis were selected, having 300 CT images labeled as Normal.

\section{DATA AUGMENTATION}

A large number of training images is required to train deep learning models, especially for image classification tasks. When only a small number of training images is available, model overfitting to the training images arises, which weakens the model's ability to adapt to new data. Different image augmentation techniques exist that can be used to improve the performance and generalization of deep learning models. These techniques rely on the creation of different forms of the original images used for training [36].
In our case, five augmentation techniques were used in the training of all models, including random reflection in both horizontal and vertical axes. The horizontal reflection applies the random reflection in the left-right direction of the image, while the vertical reflection applies the random reflection in the top-bottom direction. The other two techniques used were image translation on both directions of the input image, including the $\mathrm{x}$-axis direction (horizontally) and the $\mathrm{y}$-axis direction (vertically). It is necessary to specify a pixel range for this translation technique, which was set at 10 pixels for both translation directions. The fifth augmentation technique was random rotation, which was set in the range of $10^{\circ}$ image rotation clockwise and anticlockwise.

\section{EXPERIMENTS AND RESULTS}

All experiments were performed on a machine running the 64-bit Windows 10 operating system. The machine had an Intel ${ }^{\circledR}$ Core $^{\mathrm{TM}}$ i7-8750H CPU @ 2.20GHz and 32GB of RAM, and it was equipped with an NVIDIA GeForce 1070 with 8GB of GPU memory. Model training and testing were implemented and evaluated using the 64-bit version of MATLAB R2020b.

To increase the generalization and reliability of the results, as well as due to data limitations, 5-fold cross-validation was used to validate the trained models. Depending on the type of model and its dataset, as in Table I, each model was trained and tested five times; every time, one-fold was used for testing, while the other four folds were used for training and validation. The data splitting approach was $70 \%, 10 \%$, and $20 \%$ for training, validation, and testing, respectively.

TABLE I. DATASET PREPARATION FOR EACH MODEL

\begin{tabular}{|c|c|c|c|c|}
\hline Model & $\begin{array}{l}\text { Labeling } \\
\text { Type }\end{array}$ & $\begin{array}{l}\text { Labeling } \\
\text { As }\end{array}$ & $\begin{array}{l}\text { No. } \\
\text { Images }\end{array}$ & $\begin{array}{l}\text { No. } \\
\text { Patients }\end{array}$ \\
\hline \multirow{2}{*}{$\begin{array}{l}\text { Brain CT Slice } \\
\text { Classification }\end{array}$} & \multirow{2}{*}{$\begin{array}{l}\text { Image } \\
\text { as a class }\end{array}$} & Brain CT Slice & 570 & 50 \\
\hline & & Not Brain CT Slice & 560 & 50 \\
\hline \multirow{2}{*}{$\begin{array}{l}\text { Brain Tissue } \\
\text { Segmentation }\end{array}$} & \multirow{2}{*}{$\begin{array}{l}\text { Pixel } \\
\text { as a class }\end{array}$} & Brain Tissue & \multirow{2}{*}{365} & \multirow{2}{*}{18} \\
\hline & & Not Brain Tissue & & \\
\hline \multirow{2}{*}{$\begin{array}{l}\text { Brain Tissue } \\
\text { Classification }\end{array}$} & \multirow{2}{*}{$\begin{array}{l}\text { Image } \\
\text { as a class }\end{array}$} & Acute & 300 & 63 \\
\hline & & Normal & 300 & 62 \\
\hline
\end{tabular}

In the Brain CT Slice Classification Model, 5-fold crossvalidation was used to evaluate each of the fine-tuned pretrained models (i.e., AlexNet, GoogleNet, ResNet18, and ResNet50). In each run of this approach, 226 images were used as a testing fold, while from the remaining images, 814 and 90 images were used for training and validation, respectively. For the Brain Tissue Segmentation Model, the adjustments to the pre-trained models, which included updating their structures to match the FCN-8 structure, were also evaluated using 5-fold cross-validation. In this case, each fold included 263, 29, and 73 images for training, validation, and testing, respectively.

Evaluation of the Brain Tissue Classification Model included evaluating the classification performance of the finetuned pre-trained models as well as the proposed 5S-CNN model in slice-level and patient-level classifications. Slicelevel evaluation, considering that the same patient's slices are in only the training, validation, or testing dataset, demonstrates the slice-wise classification performance. However, patient- 
level classification analyzes the performance for every slice of a patient and reports a single resulting class for that patient. Although patient-wise evaluation is the standard approach in the medical field [37], slice-level evaluation was also performed because a patient should be diagnosed with ischemic stroke if at least one slice of the patient's CT scan shows a sign of the ischemic stroke. The slice-level dataset splitting approach does not strictly comply with the $70 \%, 10 \%$, and $20 \%$ method, especially when considering the use of crossvalidation. The reason is that every patient will have a different number of CT slices that are affected by ischemic stroke. The patient-level acute vs. normal classification included 125 patients, and it was validated using 5-fold cross-validation. In each run of this approach, 25 patients were used as a testing fold, while from the remaining 100 patients, 90 were used for training and 10 for validation. ELEHE contrast enhancement was applied to each input image prior to Brain Tissue Classification.

Among the available optimization algorithms, the Adam optimization algorithm has been shown to outperform its counterparts. Therefore, all trained models were trained using the Adam optimization algorithm with a gradient decay factor of 0.9. The initial learning rate was set to 0.001 while the regularization factor was set to 0.0001 . Each model was trained for 100 epochs with a minibatch size of 16 due to memory limitations.

For evaluation, the commonly employed performance measurements of accuracy, sensitivity, specificity, precision, and F1 score are used. However, the performance of segmentation models was evaluated in terms of pixel global accuracy, mean recall, mean Intersection over Union (IoU), Weighted Intersection Over Union (wIoU), and mean Boundary F1 (BF) score. Using 5-fold cross-validation, the mean and standard deviation of the resulting measurements over the five testing folds were reported. Performance measurements of the classification models were derived based on the concepts of True Positive (TP), True Negative (TN), False Positive (FP), and False Negative (FN), as in (2-7).

For the evaluation of segmentation models, the pixel global accuracy measurement indicates the percentage of correctly identified pixels corresponding to the total number of pixels, regardless of the class type, as in (2). Mean recall measures the ratio of accurately classified pixels to the total number of pixels based on class type, which is averaged across both classes (see (3)). IoU computes the ratio of accurately classified pixels to the total number of ground truth and predicted pixels based on class type, as shown in (7). By averaging the resulting IoU over classes, the mean IoU can be obtained. In wIoU, the average IoU of each class is weighted by the total number of pixels in its corresponding class. The BF score calculates the predicted boundary of each class relative to its true boundary, as shown in (6). The mean BF score can then be obtained by averaging the resulting $\mathrm{BF}$ scores over classes.

$$
\begin{aligned}
& \text { Accuracy }=\frac{T P+T N}{T P+T N+F P+F N}(\times 100 \%) \\
& \text { Sensitivity }=\frac{F P+F N}{T P+T N+F P+F N}(\times 100 \%) \\
& \text { Specificity }=\frac{T P}{T P+F N}(\times 100 \%)
\end{aligned}
$$

$$
\begin{aligned}
& \text { Precision }=\frac{T N}{F P+T N}(\times 100 \%) \\
& F 1 \text { Score }=\frac{T P}{T P+F P}(\times 100 \%) \\
& \mathrm{IoU}=\frac{2 T P}{2 T P+F P+F N}(\times 100 \%)
\end{aligned}
$$

\section{A. Experimental Results for Brain CT Slice Classification}

The experimental results for the brain CT slice classification task, which is the first step of the proposed methodology (as shown in Fig. 3), are given in Table II. Each in Table II presents the evaluation results for each of the finetuned pre-trained CNN models (i.e., AlexNet, GoogleNet, ResNet18, and ResNet50). For each evaluated model, the mean and standard deviation of the resulting measurements are computed using 5-fold cross-validation.

Based on the obtained results (see Table II), the selected model for the brain CT slice classification task was the finetuned ResNet50. This decision was made because ResNet50outperformed other models on each metric except sensitivity which was equal to ResNet18 but better in terms of standard deviation.

\section{B. Experimental Results for Brain Tissue Segmentation}

The brain tissue segmentation results, using FCN-8 with different fine-tuned backbone models, are shown in Table III. In this experiment, 365 images were used, in which every pixel within each image was labeled as either a brain tissue pixel or not a brain tissue pixel. The results of Table III show that both ResNet18 and ResNet50 produced similar results, but both outperformed other models.

FCN-8 with fine-tuned ResNet50 performed excellently in segmenting brain tissue pixels, achieving 99.70\% global accuracy with a standard deviation of $0.04 \%$ (see Table III). However, certain pixels were misclassified. Therefore, as explained in section II, post-processing was applied after brain tissue segmentation; the area of the largest segmented connected group of pixels in the CT slice (which usually represents brain tissue) was compared to a threshold fixed at 1,000 pixels to either include or exclude the brain CT slice for further processing. The threshold was fixed based on the recommendations of the medical team. This comparison ensures that the area of the segmented brain tissue is sufficiently large for the subsequent brain tissue classification task. After this, it is necessary to fill the holes within the segmented brain tissue to ensure that misclassified brain tissue pixels are included in the largest segmented connected group of pixels. Fig. 10 shows experimental examples of segmented brain tissues using FCN-8 with fine-tuned ResNet50 followed by post-processing.

\section{Experimental Results for Brain Tissue Classification}

After brain tissue segmentation, the final task is to classify the segmented brain tissue as ischemic stroke or normal. Patient-wise and slice-wise classification experiments were performed for validation in this experiment. Each input image was enhanced using the ELEHE contrast enhancement technique prior to the training and testing of the fine-tuned pretrained models and the 5S-CNN. As shown in Table IV, the 5S-CNN model with ELEHE contrast enhancement 
outperformed the other models for every scenario, reflecting its power in terms of multi-scale feature learning. In addition, the decision made regarding the number of branches used in the proposed multi-scale CNN model (5S-CNN) was based on experimenting with the multi-scales of 2, 3, 4, and 5 branches. Five branches were found to yield the best results in all scenarios (patient-wise and slice-wise classification), as shown in Table $\mathrm{V}$.

TABLE II. BRAin CT SliCE ClassifiCATION RESUlts (MEAN \pm STANDARD DeVIATION) AVERAGE OVER THE FIVE Folds OF EACH Model

\begin{tabular}{|c|c|c|c|c|c|}
\hline Model & Accuracy & Sensitivity & Specificity & Precision & F1 Score \\
\hline AlexNet & $98.31 \pm 0.63$ & $98.33 \pm 0.47$ & $97.01 \pm 1.45$ & $97.99 \pm 0.67$ & $98.79 \pm 0.53$ \\
\hline GoogleNet & $98.59 \pm 0.41$ & $98.80 \pm 0.48$ & $98.17 \pm 0.74$ & $98.21 \pm 0.45$ & $98.95 \pm 0.65$ \\
\hline ResNet18 & $99.04 \pm 0.38$ & $99.17 \pm 0.88$ & $98.92 \pm 1.24$ & $98.94 \pm 1.20$ & $99.04 \pm 0.37$ \\
\hline ResNet50 & $99.21 \pm 0.31$ & $99.17 \pm 0.42$ & $99.25 \pm 0.68$ & $99.25 \pm 0.68$ & $99.21 \pm 0.31$ \\
\hline
\end{tabular}

TABLE III. BRAin TISSUE SEgMENTATION RESUlts (MEAN \pm STANDARD DEVIATION) AVERAGE OVER THE FIVE FOLDS OF EACH MOdEL

\begin{tabular}{|c|c|c|c|c|c|}
\hline Model & Global Accuracy & Mean Sensitivity & Mean IoU & Weighted IoU & Mean F1 Score \\
\hline AlexNet & $97.97 \pm 2.60$ & $98.43 \pm 1.91$ & $94.41 \pm 6.81$ & $96.32 \pm 4.43$ & $87.44 \pm 13.69$ \\
\hline GoogleNet & $97.58 \pm 3.28$ & $98.23 \pm 1.87$ & $93.58 \pm 8.04$ & $95.72 \pm 5.41$ & $84.44 \pm 19.43$ \\
\hline ResNet18 & $99.63 \pm 0.08$ & $99.69 \pm 0.04$ & $98.85 \pm 0.28$ & $99.26 \pm 0.15$ & $98.87 \pm 0.50$ \\
\hline ResNet50 & $99.70 \pm 0.04$ & $99.75 \pm 0.04$ & $99.06 \pm 0.15$ & $99.40 \pm 0.08$ & $98.93 \pm 0.18$ \\
\hline
\end{tabular}
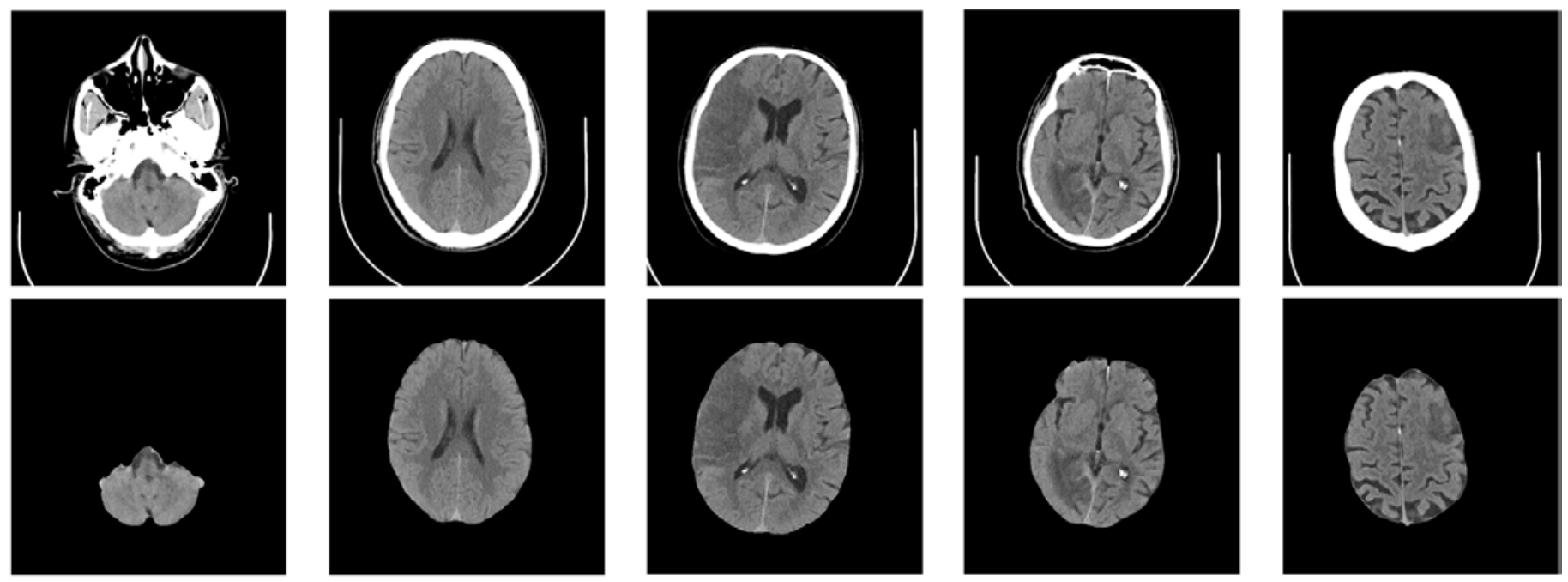

Fig. 10. Examples of Segmented Brain Tissues using FCN-8 with Fine-Tuned ResNet50 Followed by Post-processing: Original Image (Top), Segmented Image (Bottom).

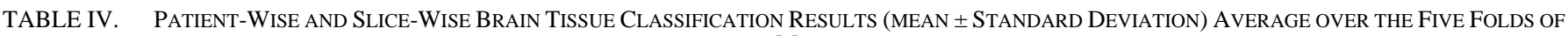
EACH MODEL

\begin{tabular}{|l|l|l|l|l|l|l|}
\hline Scenario & Model & Accuracy & Sensitivity & Specificity & Precision & F1 Score \\
\hline \multirow{4}{*}{$\begin{array}{l}\text { Patient-wise } \\
\text { classification }\end{array}$} & AlexNet & $74.40 \pm 8.29$ & $84.57 \pm 4.95$ & $70.27 \pm 9.25$ & $56.67 \pm 17.08$ & $66.95 \pm 12.94$ \\
\cline { 2 - 7 } & GoogleNet & $75.20 \pm 7.16$ & $81.95 \pm 11.07$ & $74.36 \pm 10.11$ & $65.00 \pm 22.36$ & $69.80 \pm 14.03$ \\
\cline { 2 - 7 } & ResNet18 & $79.20 \pm 6.57$ & $82.98 \pm 6.39$ & $77.59 \pm 9.10$ & $71.67 \pm 13.94$ & $76.30 \pm 8.68$ \\
\cline { 2 - 7 } & ResNet50 & $81.60 \pm 6.69$ & $86.79 \pm 7.58$ & $79.35 \pm 8.91$ & $73.33 \pm 14.91$ & $78.68 \pm 9.72$ \\
\cline { 2 - 7 } & $\mathbf{5 S - C N N}$ & $\mathbf{8 7 . 2 0} \pm \mathbf{5 . 9 3}$ & $\mathbf{8 8 . 9 5} \pm \mathbf{3 . 6 7}$ & $\mathbf{8 7 . 4 4} \pm \mathbf{8 . 8 9}$ & $\mathbf{8 5 . 0 0} \pm \mathbf{1 2 . 3 6}$ & $\mathbf{8 6 . 1 9} \pm \mathbf{6 . 6 5}$ \\
\hline \multirow{4}{*}{$\begin{array}{l}\text { Slice-wise } \\
\text { classification }\end{array}$} & AlexNet & $76.61 \pm 6.34$ & $78.11 \pm 7.66$ & $76.43 \pm 12.17$ & $70.73 \pm 16.52$ & $73.39 \pm 9.04$ \\
\cline { 2 - 7 } & GoogleNet & $79.30 \pm 6.84$ & $80.37 \pm 8.35$ & $79.09 \pm 10.00$ & $77.82 \pm 9.87$ & $78.57 \pm 6.42$ \\
\cline { 2 - 7 } & ResNet18 & $82.61 \pm 6.27$ & $80.44 \pm 5.79$ & $85.50 \pm 12.93$ & $84.35 \pm 15.78$ & $81.40 \pm 6.68$ \\
\cline { 2 - 7 } & ResNet50 & $84.14 \pm 4.09$ & $83.18 \pm 6.01$ & $85.34 \pm \mathbf{5 . 3 9}$ & $85.08 \pm \mathbf{5 . 0 4}$ & $83.96 \pm 3.81$ \\
\cline { 2 - 7 } & $\mathbf{5 S - C N N}$ & $\mathbf{9 0 . 5 1} \pm \mathbf{2 . 2 2}$ & $\mathbf{9 0 . 7 2} \pm \mathbf{5 . 3 8}$ & $\mathbf{9 1 . 3 8} \pm 5.71$ & $\mathbf{9 0 . 1 5} \pm 7.77$ & $\mathbf{9 0 . 1 1} \pm \mathbf{2 . 7 8}$ \\
\hline
\end{tabular}


TABLE V. ImPact of Scales on the Proposed Multi-Scale CNN For Patient-Wise AND Slice-Wise Classifications (MEAN \pm Standard DEVIATION) AVERAGE OVER THE FIVE FOLDS OF EACH MODEL

\begin{tabular}{|l|l|l|l|l|l|l|}
\hline Scenario & Model & Accuracy & Sensitivity & Specificity & Precision & F1 Score \\
\hline \multirow{4}{*}{$\begin{array}{l}\text { Patient-wise } \\
\text { classification }\end{array}$} & 2S-CNN & $73.60 \pm 6.07$ & $80.23 \pm 7.71$ & $71.90 \pm \mathbf{8 . 4 5}$ & $61.67 \pm 19.18$ & $67.81 \pm 12.31$ \\
\cline { 2 - 7 } & SS-CNN & $71.20 \pm 11.10$ & $80.42 \pm \mathbf{5 . 6 3}$ & $68.82 \pm 13.75$ & $51.67 \pm 25.95$ & $60.51 \pm 19.73$ \\
\cline { 2 - 7 } & SS-CNN & $78.40 \pm 6.69$ & $78.36 \pm 8.15$ & $79.59 \pm 8.68$ & $76.67 \pm 13.36$ & $77.05 \pm 8.08$ \\
\cline { 2 - 7 } & $\mathbf{5 S - C N N}$ & $\mathbf{8 7 . 2 0} \pm \mathbf{5 . 9 3}$ & $\mathbf{8 8 . 9 5} \pm 8.67$ & $\mathbf{8 7 . 4 4} \pm 8.89$ & $\mathbf{8 5 . 0 0} \pm \mathbf{1 2 . 3 6}$ & $\mathbf{8 6 . 1 9} \pm \mathbf{6 . 6 5}$ \\
\hline \multirow{4}{*}{$\begin{array}{l}\text { Slice-wise } \\
\text { classification }\end{array}$} & 2S-CNN & $83.42 \pm 4.73$ & $86.46 \pm \mathbf{3 . 5 7}$ & $81.45 \pm 8.15$ & $78.82 \pm 9.60$ & $82.11 \pm 5.07$ \\
\cline { 2 - 7 } & 3S-CNN & $82.44 \pm 5.65$ & $84.47 \pm 9.43$ & $82.08 \pm 10.49$ & $79.91 \pm 13.25$ & $81.19 \pm 6.83$ \\
\cline { 2 - 7 } & 4S-CNN & $86.34 \pm 3.29$ & $88.08 \pm 4.24$ & $85.27 \pm 7.32$ & $83.48 \pm 9.59$ & $85.35 \pm 4.55$ \\
\cline { 2 - 7 } & SS-CNN & $\mathbf{9 0 . 5 1} \pm \mathbf{2 . 2 2}$ & $\mathbf{9 0 . 7 2} \pm 5.38$ & $\mathbf{9 1 . 3 8} \pm \mathbf{5 . 7 1}$ & $\mathbf{9 0 . 1 5} \pm \mathbf{7 . 7 7}$ & $\mathbf{9 0 . 1 1} \pm \mathbf{2 . 7 8}$ \\
\hline
\end{tabular}

\section{DISCUSSION}

The first two tasks (i.e., CT slice classification and brain region segmentation) are relatively easy problems; ResNet50 and FCN-8 based on ResNet50 work adequately in both cases. However, the third task (i.e., classification of brain tissue as normal or ischemic stroke) is comparatively difficult due to the textural similarity between the normal region and the region affected by ischemic stroke. For this purpose, the 5S-CNN model is proposed. In this section, we discuss the classification results for fine-tuned pre-trained networks as well as the proposed 5S-CNN regarding the task of classifying ischemic stroke against normal cases in patient-wise and slice-wise classifications, the results for which are given in Table IV.

In brain tissue classification, AlexNet and GoogleNet achieved the lowest performance in both patient-wise and slice-wise classifications. Mean accuracies of $74.40 \%$ and $75.20 \%$ and standard deviations of $8.29 \%$ and $7.16 \%$ resulted from both fine-tuned models in patient-wise classification, while slice-wise classification achieved mean accuracies of $76.61 \%$ and $79.30 \%$ and standard deviations of $6.34 \%$ and $6.84 \%$. For patient-wise classification using AlexNet, the values for average sensitivity, specificity, precision, and F1 score were $84.57 \%, 70.27 \%, 56.67 \%$, and $66.95 \%$, respectively, while GoogleNet achieved 81.95\%, 74.36\%, 65\%, and 69.80\% for these metrics. However, slice-wise classification resulted in an average sensitivity of $78.11 \%$, specificity of $76.43 \%$, precision of $70.73 \%$, and F1-score of $73.39 \%$, while GoogleNet achieved 80.37\%, 79.09\%, 77.82\%, and 78.57\% for these metrics, respectively. The standard deviation of sensitivity for AlexNet was $4.95 \%$ in the patient-wise scenario and $7.66 \%$ in the slice-wise scenario, while for GoogleNet, the values were $11.07 \%$ and $8.35 \%$, respectively. Notably, the accuracy difference of $2.69 \%$ in the patient-wise scenario and $0.8 \%$ in the slice-wise scenario indicates the favorable generalization performance of GoogleNet. Compared to GoogleNet, ResNet18 and ResNet50 were associated with better performance, achieving mean accuracies of $79.20 \%$ and $81.60 \%$ in patient-wise classification and $82.61 \%$ and $84.14 \%$ in slice-wise classification, respectively.

For ResNet18, the average values for sensitivity, specificity, precision, and F1 score were $82.98 \%, 77.59 \%, 71.67 \%$, and $76.30 \%$, respectively in the patient-wise scenario, while the values for the same metrics were $80.44 \%$, $85.50 \%$, $84.35 \%$, and $81.40 \%$ in the slice-wise scenario. ResNet50 yielded better performance values with an average sensitivity of $86.79 \%$, specificity of $79.35 \%$, precision of $73.33 \%$, and F1 score of $78.68 \%$ in the patient-wise scenario, while the slice-wise scenario resulted in $83.18 \%, 85.34 \%, 85.08 \%$, and $83.69 \%$, respectively. Only in the slice-wise scenario, ResNet50 outperformed ResNet18 in all performance metrics except specificity, where the results indicated a specificity difference of $0.16 \%$ for ResNet18.

The proposed model, 5S-CNN, outperformed the other models on every metric in both patient-level and slice-level classification. Values of $87.20 \%$, 88.95\%, 87.44\%, 85.00\%, and $86.19 \%$ were achieved in the patient-wise scenario with respect to sensitivity, specificity, precision, and F1 score, while values of $90.51 \%$, 90.72\%, 91.38\%, 91.15\%, and 90.11\% were achieved for slice-wise classification. Also, the standard deviation of the proposed model outperformed the other models on all metrics except specificity and precision, only in the slice-wise scenario. In this case, the standard deviation of 5S-CNN was $5.71 \%$ and $7.77 \%$ in these two metrics, whereas the specificity and precision of ResNet50 were $5.39 \%$ and $5.04 \%$, respectively. Acute stroke is an early sign of ischemic stroke, and it is very difficult to identify due to the subtle differences that exist between normal brain regions and those affected by acute ischemic stroke. However, in this case, 5SCNN yielded an excellent performance overall.

Table VI shows a comparison of model complexity for the ischemic stroke classification models based on the number of parameters and Floating-Point Operations (FLOPs). The number of parameters is the sum of all learnable weights and biases of all Conv and FC layers within a CNN model. By contrast, the number of FLOPs reflects the computations required for a single forward pass within the model. After adopting the pre-trained networks for brain CT images, AlexNet had the greatest number of parameters (approximately 57.7 million) and around 7.6 billion FLOPs. GoogleNet consisted of approximately 5.1 million parameters and 7.7 billion FLOPs. Notably, despite the substantial difference in the number of parameters, AlexNet and GoogleNet had almost the same number of FLOPs. This is attributable to the small kernel sizes and feature maps of GoogleNet, which contributed to the similar computational cost of AlexNet and GoogleNet. ResNet18 had approximately 11.2 million parameters and 9.1 billion FLOPs, meaning that its computational cost exceeds AlexNet and GoogleNet. ResNet50 had the largest number of FLOPs compared to the other models, amounting to 
approximately 19.8 billion FLOPs, along with 23.2 million parameters. The significant number of FLOPs means that ResNet50 is substantially more computationally expensive and consumes more training time compared to its counterparts. The proposed model, 5S-CNN, has the lowest number of parameters (approximately 0.8 million) and FLOPs (approximately 0.6 billion). Therefore, in addition to outperforming the other models in terms of classification results, 5S-CNN is also more computationally efficient.

TABLE VI. COMPLEXITY OF MODELS USED FOR STROKE CLASSIFICATION

\begin{tabular}{|l|l|l|}
\hline Model & $\begin{array}{l}\text { Parameters } \\
\text { (Millions) }\end{array}$ & $\begin{array}{l}\text { FLOPs } \\
\text { (Billions) }\end{array}$ \\
\hline AlexNet & 57.5 & 7.6 \\
\hline GoogleNet & 5.1 & 7.7 \\
\hline ResNet18 & 11.2 & 9.1 \\
\hline ResNet50 & 23.5 & 19.8 \\
\hline 5S-CNN & $\mathbf{0 . 8}$ & $\mathbf{0 . 6}$ \\
\hline
\end{tabular}

It is worth comparing the proposed method to those of Pereira et al. [15], Anis et al. [16], and Gautam et al. [17]. Each of these researchers also leveraged DL methods for ischemic stroke detection using brain CT images. The performance of these methods, as reported in the introduction, was determined based on each research group's private datasets. Therefore, to facilitate a fair comparison, we implemented these methods and trained them on the collected dataset based on the authors' recommendations. Both patient-wise and slice-wise brain tissue classification experiments were performed. The results and confusion matrices are shown in Table VII, as well as Fig. 11.

For the classification of acute ischemic stroke against normal cases, the results in Table VII show that 5S-CNN outperformed the other three methods on all performance metrics in both the patient-wise and slice-wise scenarios. Furthermore, the confusion matrices in Fig. 11(a) show the decisions that each method made regarding the optimal testing fold across the five cross-validated folds. In this fold, a total of 25 patients with 136 CT images were used for testing. Among those, 13 patients with 73 images had acute ischemic stroke, while 63 images were normal from the remaining normal patients.

In the patient-wise scenario, 5S-CNN correctly classified all acute patients as having acute ischemic stroke, whereas the other methods misclassified at least one acute patient as a normal patient. In this case, the classifier determines that a patient has acute ischemic stroke if one of the patient's CT slices is classified as having acute stroke. Due to this, the methods of Pereira et al. [15] and Anis et al. [16] were able to correctly classify 4 normal patients, while 8 were misclassified as having acute ischemic stroke. Also, the method proposed by
Gautam et al. [17] misclassified 9 normal patients as having acute ischemic stroke. In the case of 5S-CNN, only 2 normal patients were misclassified as having acute ischemic stroke. As such, 5S-CNN outperformed all other models in this area.

A similar trend is seen in the scenario of slice-wise classification, as shown in Fig. 11(b). 5S-CNN correctly classified 69 acute stroke slices out of 73 images, while 21, 11, and 38 images were misclassified in Pereira et al. [15], Anis et al. [16], and Gautam et al. [17], respectively. In the classification of normal images, the compared methods misclassified more than 14 normal images as acute ischemic stroke, while 5S-CNN misclassified only 6 images out of the 63 normal tested images. Although our model misclassified 4 images as normal from the acute patients in slice-wise classification, it correctly classified all patients as suffering from acute ischemic stroke in the patient-wise scenario. The encoding of multi-scale information from the CT scans proves the potential of the $5 \mathrm{~S}-\mathrm{CNN}$ model to improve clinical decision-making.

Compared to state-of-the-art models, as well as similar works, the proposed method uses a fully automated approach to analyze brain CT images and determine whether it includes brain tissue or not. In turn, through the segmentation of brain tissue within the CT image, the method enables irrelevant objects and background to be eliminated, as well as ensuring the accurate segmentation of all brain tissue pixels using morphological operations. Following this, the method applies the ELEHE contrast enhancement technique to boost the interior details of the segmented brain tissue, thereby facilitating more effective classification. Finally, classification of the resulting brain tissue using the proposed model outperformed state-of-the-art CNN models, as well as models proposed in similar previous methods. All CT slices in the collected dataset were used without altering their original 16bit greyscale range, thereby ensuring the stability of the original pixel values. Additionally, rather than resizing original images to fit the pre-trained networks, the pre-trained networks were fine-tuned to fit the original size of CT brain images. Taken together, the use of 5-fold cross-validation shows the generalization of the proposed 5S-CNN model, as well as its low susceptibility to overfitting.

Nevertheless, the 5S-CNN model is limited to deciding whether a brain tissue shows any signs of ischemic stroke without any localization of the stroke lesion within the brain CT slice. Another limitation of the proposed model is the possibility of misdiagnosing ischemic stroke in slices with three brain tissue areas separated by bones, which usually occur at the lower part of a brain CT scan. The developed segmentation module preserves the largest brain tissue area for further analysis and removes the rest. This decision was taken based on the medical team's recommendation for the current study, and it is a potential area for investigation in future works. 
TABLE II. COMPARISON TO RELATED METHODS APPLIED ON THE COLLECTED DATASET IN PATIENT-WISE AND SLICE-WISE CLASSIFICATIONS (MEAN \pm STANDARD DEVIATION) AVERAGE OVER THE FIVE FOLDS OF EACH MODEL

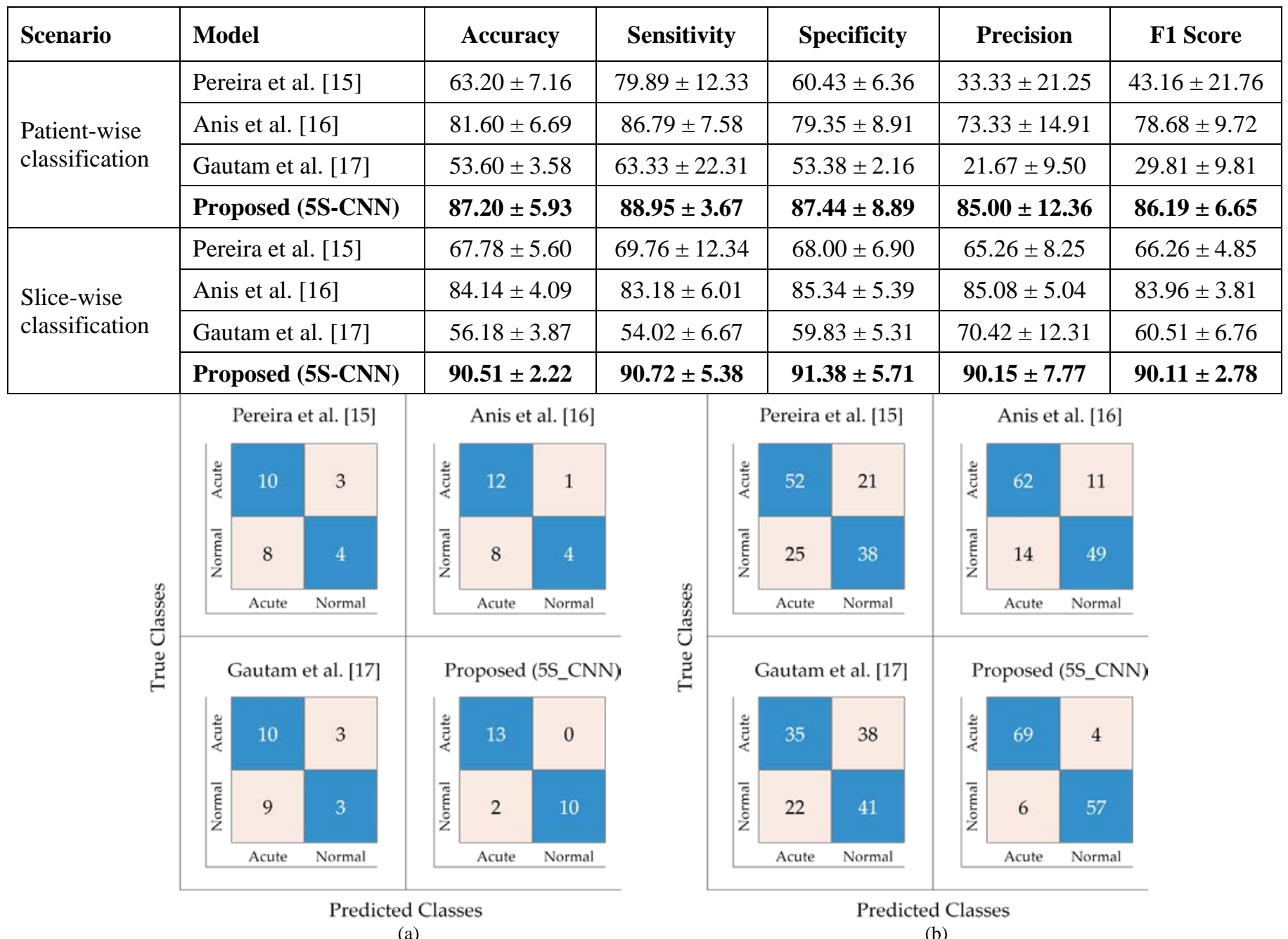

Fig. 11. Confusion Matrices of the Optimal Testing Fold from the Five Cross-Validated Folds: (a) Patient-Wise Confusion Matrices; (b) Slice-Wise Confusion Matrices.

\section{CONCLUSION}

This paper proposed a novel, automated approach for acute ischemic stroke classification in brain CT images. A preprocessing technique that can be applied to the CT scans of patients with suspected ischemic stroke was presented, including the removal of CT slices that do not contain brain tissue, segmentation of brain tissue within the remaining slices, ELEHE contrast enhancement of the segmented brain tissue, and classification of brain tissue as an ischemic stroke or normal. A lightweight multi-scale CNN model (5S-CNN) was proposed for brain tissue classification on CT slices, to determine whether the patient is experiencing an ischemic stroke. Notably, this novel model outperformed state-of-the-art models. The model uses a 5-branch architecture, with different filter sizes for each branch, to learn features at different scales, which is crucial because ischemic stroke can have any regional size and appear at any location within brain tissue. A comparison with similar methods revealed that the proposed method outperforms the best-known current methods. The main focus of this research is to identify the presence of acute ischemic stroke on CT slices automatically. However, stroke lesion segmentation is essential for treatment decisions and management. We intend to investigate this area further in the future, ideally exploiting an expansion in the size of the existing collected datasets and samples.

\section{ACKNOWLEDGMENT}

The authors are thankful to the Deanship of Scientific Research at King Saud University, Riyadh, Saudi Arabia for funding this work through the research group no. RGP-1439067. We would also like to thank Dr. Shanker Raja from King Fahad Medical City (KFMC) for his support during this research, as well as Dr. Abdulaziz AlSaad, Dr. Juman AlGhamdi, and Dr. Abeer AlDhawi from the Neurology Department at KFMC. 


\section{REFERENCES}

[1] World Health Organization, "The top 10 causes of death," 2019. [Online]. Available: https:/www.who.int/news-room/fact-sheets/detail/the-top-10causes-of-death.

[2] N. H. Rajini and R. Bhavani, "Computer aided detection of ischemic stroke using segmentation and texture features," Measurement, pp. 18651874, 2013.

[3] A. R. Asirvatham and M. Z. Marwan, "Stroke in Saudi Arabia: a review of the recent literature," Pan African Medical Journal, vol. 17, no. 1, 2014.

[4] F. Al-Senani, M. Al-Johani, M. Salawati, A. Alhazzani, L. B. Morgenstern, V. S. Ravest, M. Cuche and S. Eggington, "An Epidemiological Model for First Stroke in Saudi Arabia," Journal of Stroke and Cerebrovascular Diseases, vol. 29, no. 1, p. 104465, 2020.

[5] K. K. Andersen, T. S. Olsen, C. Dehlendroff and L. P. Kammersgaard, "Hemorrhagic and Ischemic Strokes Compared: Stroke Severity, Mortality, and Risk Factors," Stroke: , pp. 2068-2072, 2009.

[6] A. R. Xavier, A. I. Qureshi, J. F. Kirmani, A. M. Yahia and R. Bakshi, "Neuroimaging of Stroke: A Review," Southern Medical Journal, vol. 96, no. 4, pp. 367-379, 2003.

[7] C. K. Hansen, A. Christensen, H. Rodgers, I. Havsteen, C. Kruuse and H. Christensen, "Does the Primary Imaging Modality-Computed Tomography or Magnetic Resonance Imaging-Influence Stroke Physicians' Certainty on Whether or Not to Give Thrombolysis to Randomized Acute Stroke Patients?," Journal of Stroke and Cerebrovascular Diseases, 2017.

[8] Z. Calic, C. Cappelen-Smith, C. S. Anderson, W. Xuan and D. J. Cordato, "Cerebellar infarction and factors associated with delayed presentation and misdiagnosis," Cerebrovascular Diseases, vol. 42, no. 5-6, pp. 476484, 2016.

[9] G. Litjens, T. Kooi, B. E. Bejnordi, A. A. A. Setio, F. Ciompi, M. Ghafoorian, J. v. d. Laak, B. v. Ginneken and C. I. Sánchez, "A survey on deep learning in medical image analysis," Medical Image Analysis, vol. 42, pp. 60-88, 2017.

[10] G. Kaur and J. Chhaterji, "A Survey on Medical Image Segmentation," International Journal of Science and Research (IJSR), vol. 6, no. 5, pp. 2319-7064, 2017.

[11] H. R. Roth, C. Shen, H. Oda, M. Oda, Y. Hayashi, K. Misawa and K. Mori, "Deep learning and its application to medical image segmentation," Medical Imaging Technology, vol. 36, no. 2, pp. 63-71, 2018.

[12] C.-M. Lo, P.-H. Hung and K. L.-C. Hsieh, "Computer-aided detection of hyperacute stroke based on relative radiomic patterns in computed tomography," Applied Sciences, vol. 9, no. 8, p. 1668, 2019.

[13] G. Wu, J. Lin, X. Chen, Z. Li, Y. Wang, J. Zhao and J. Yu, "Early identification of ischemic stroke in noncontrast computed tomography," Biomedical Signal Processing and Control, vol. 52, pp. 41-52, 2019.

[14] C.-L. Chin, B.-J. Lin, G.-R. Wu, T.-C. Weng, C.-S. Yang, R.-C. Su and Y.-J. Pan, "An Automated Early Ischemic Stroke Detection System using CNN Deep Learning Algorithm," 2017 IEEE 8th International Conference on Awareness Science and Technology (iCAST), pp. 368372, 2017.

[15] D. R. Pereira, P. P. R. Filho, G. H. d. Rosa, J. P. Papa and V. H. C. d. Albuquerque, "Stroke Lesion Detection Using Convolutional Neural Networks," 2018 International Joint Conference on Neural Networks (IJCNN), pp. 1-6, 2018.

[16] A. A. M. Suberi, W. N. W. Zakaria, R. Tomari, A. Nazari, M. N. H. Mohd and N. F. N. Fuad, "Deep Transfer Learning Application for Automated Ischemic Classification in Posterior Fossa CT Images," International Journal of Advanced Computer Science and Applications (IJACSA), vol. 10, no. 8, pp. 459-465, 2019.

[17] A. Gautam and B. Raman, "Towards effective classification of brain hemorrhagic and ischemic stroke using CNN," Biomedical Signal Processing and Control, vol. 63, p. 102178, 2021.
[18] X. Bai, Y. Zhang, F. Zhou and B. Xue, "Quadtree-based multi-focus image fusion using a weighted focus-measure," Information Fusion, vol. 22, pp. 105-118, 2015.

[19] S. Zhang, M. Zhang, S. Ma, Q. Wang, Y. Qu, Z. Sun and T. Yang, "Research Progress of Deep Learning in the Diagnosis and Prevention of Stroke," BioMed Research International, 2021.

[20] M. S. Sirsat, E. Ferme and J. Camara, "Machine Learning for Brain Stroke: A Review," Journal of Stroke and Cerebrovascular Diseases, vol. 29, no. 10, p. 105162, 2020.

[21] A. Krizhevsky, I. Sutskever and G. E. Hinton, "ImageNet Classification with Deep Convolutional Neural Networks," Communications of the ACM, vol. 60, no. 6, pp. 84-90, 2017.

[22] L. M. Allen, A. N. Hasso, J. Handwerker and H. Farid, "Sequencespecific MR Imaging Findings That Are Useful in Dating Ischemic Stroke," Radiographics, vol. 32, no. 5, pp. 1285-1297, 2012.

[23] C. Szegedy, W. Liu, Y. Jia, P. Sermanet, S. Reed, D. Anguelov, D. Erhan, V. Vanhoucke and A. Rabinovich, "Going deeper with convolutions," Proceedings of IEEE Computer Society Conference on Computer Vision and Pattern Recognition, pp. 1-9, 2015.

[24] K. H. X. Zhang, S. Ren and J. Sun, "Deep Residual Learning for Image Recognition," Proceedings of IEEE Computer Society Conference on Computer Vision and Pattern Recognition, pp. 770-778, 2016.

[25] P. Soille, Morphological Image Analysis: Principles and Applications, Springer Science \& Business Media, 2004.

[26] W. Shi and H. Liu, "Modified U-Net Architecture for Ischemic Stroke Lesion Segmentation and Detection," 2019 IEEE 4th Advanced Information Technology, Electronic and Automation Control Conference (IAEAC 2019), vol. 1, pp. 1068-1071, 2019.

[27] A. Manvel, K. Vladimir, T. Alexander and U. Dmitry, "Radiologist-Level Stroke Classification on Non-contrast CT Scans with Deep U-Net," International Conference on Medical Image Computing and ComputerAssisted Intervention, pp. 820-828, 2019.

[28] H. Kuang, B. K. Menon and W. Qiu, "Segmenting Hemorrhagic and Ischemic Infarct Simultaneously From Follow-Up Non-Contrast CT Images in Patients With Acute Ischemic Stroke," IEEE Access, vol. 7, pp. 39842-39851, 2019.

[29] J. Long, E. Shelhamer and T. Darrell, "Fully Convolutional Networks for Semantic Segmentation," Proceedings of the IEEE conference on computer vision and pattern recognition, pp. 3431-3440, 2015.

[30] R. C. Gonzalez and R. E. Woods, Digital Image Processing, Pearson Education Limited, 2018.

[31] B. Kurt, V. V. Nabiyev and K. Turhan, "Medical Images Enhancement by using Anisotropic Filter and CLAHE," 2012 International Symposium on Innovations in Intelligent Systems and Applications, pp. 1-4, 2012.

[32] S.-C. Huang, F.-C. Cheng and Y.-S. Chiu, "Efficient Contrast Enhancement Using Adaptive Gamma Correction With Weighting Distribution," IEEE TRANSACTIONS ON IMAGE PROCESSING, vol. 22, no. 3, pp. 1032-1041, 2013.

[33] Z. Al-Ameen and G. Sulong, "A New Algorithm for Improving the Low Contrast of Computed Tomography Images Using Tuned Brightness Controlled Single-Scale Retinex," Scanning, vol. 37, no. 2, pp. 116-125, 2015.

[34] T. V, K. S. Sim and E. K. Wong, "Brain Early Infarct Detection Using Gamma Correction Extreme-Level Eliminating With Weighting Distribution," Scanning, vol. 38, no. 6, pp. 842-856, 2016.

[35] T.-L. Tan, K.-S. Sim and A.-K. Chong, "Contrast Enhancement of CT Brain Images for Detection of Ischemic Stroke," 2012 International Conference on Biomedical Engineering (ICoBE), pp. 385-388, 2012.

[36] C. Shorten and T. M. Khoshgoftaar, "A survey on Image Data Augmentation," Journal of Big Data, vol. 6, no. 1, pp. 1-48, 2019.

[37] M. J. Willemink, W. A. Koszek, C. Hardell, J. Wu, D. Fleischmann, H. Harvey, L. R. Folio, R. M. Summers, D. L. Rubin and M. P. Lungren, "Preparing Medical Imaging Data for Machine Learning," Radiology, vol. 295, no. 1, pp. 4-15, 2020. 